\title{
Review finds better endodontic surgery outcomes with microscope use
}

\author{
Abstracted from \\ Setzer FC, Shah SB, Kohli MR, Karabucak B, Kim S. \\ Outcome of endodontic surgery: a meta-analysis of the literature-part 1: \\ Comparison of traditional root-end surgery and endodontic microsurgery. \\ J Endod 2010; 36: 1757-1765. Epub 2010 Sep 17. Review. PubMed PMID: 20951283 \\ Address for correspondence: Frank C Setzer, DMD, PhD, MS, Instructor, \\ Department of Endodontics, School of Dental Medicine, University of Pennsylvania, \\ 240 S 40th St, Philadelphia, PA 19104, USA. E-mail: fsetzer@dental.upenn.edu
}

\section{Question: In patients requiring endodontic surgery does endodontic microsurgery (EMS) result in better outcomes than traditional root-end surgery (TRS)?}

Data sources Medline, Embase and PubMed databases were searched together with hand-searches of a range of journals (Journal of Endodontics, International Endodontic Journal, Oral Surgery Oral Medicine Oral Pathology Oral Radiology and Endodontics, Journal of Oral and Maxillofacial Surgery, International Journal of Oral and Maxillofacial Surgery).

Study selection Clinical studies in several languages (English, French, German, Italian and Spanish) with a minimum follow-up of six months, evaluated using clinical and radiographic examination were included. Data extraction and synthesis Assessment and data abstraction were carried out independently. Weighted pooled success rates and relative risk assessment between TRS and EMS were calculated and a meta-analysis carried out using a random effects model.

Results Twenty-one studies were included. Weighted pooled success rates calculated from extracted raw data showed a $59 \%$ positive outcome for TRS (95\% confidence interval, $0.55-0.6308$ ) and $94 \%$ for EMS (95\% confidence interval, 0.8889-0.9816). This difference was statistically significant $(\mathrm{P}<.0005)$. The relative risk ratio showed that the probability of success for EMS was 1.58 times the probability of success for TRS

Conclusions On the basis of the meta-analysis presented, the probability of success for EMS proved significantly greater than the probability of success for TRS.

\section{Commentary}

Endodontic surgery, more commonly known as apicectomy, and root-end filling have always been considered a last resort for failed endodontic treatment. The traditional root-end surgery (TRS) using surgical burs, with amalgam for the root-end filling, was considered the gold standard for such treatment until the 1990s. The development of endodontic microsurgery (EMS), utilising the microscope, micro-instruments, ultrasonic tips and more biologically acceptable root-end filling materials, has revolutionised the procedure. The purpose of this systematic review was to evaluate the probability of success of TRS vs. EMS. ${ }^{1}$
A search of the literature produced a total of 21 studies, including seven randomised control trials, for meta-analysis. Languages were limited to English, French, German, Italian and Spanish. Although the results showed a statistically significant probability of success for EMS over TRS, one is left with a number of questions. Do the results hold true for both single rooted and multirooted teeth? Were there enough randomised control trials of large enough population to eliminate the great variability encountered in endodontic surgery?

The systematic review followed proper protocol, and its exclusion criteria eliminated some of the variability encountered in endodontic surgery. Root-end surgery performed on lesions larger than $10 \mathrm{~mm}$ and re-surgery cases were not included. Cases with less than six months of follow-up were also not included. However, one of the main variables that could affect success, the number of roots per tooth, did not seem to be taken into account; the more roots per tooth, the more chance for failure. Without knowing how many failures were for molars vs. anterior teeth, it would seem difficult to assess how truly successful EMS is vs. TRS. The authors themselves noted the need for more randomised control trials and a larger population. Perhaps tapping into other language studies would be helpful.

The ability to see better, the use of smaller instruments and more biocompatible filling materials appear to make EMS the more logical technique. However, it is difficult to tell from the study if this holds true for all types of teeth. The results showed the necessity for large-scale randomised clinical trials to enable the practitioner to have greater confidence in his evidence-based decision.

It would appear from the study that the ability to see better, the use of smaller instruments and the use of more biocompatible and better lasting filling materials would make Endodontic Microscopic Surgery a more successful procedure than Traditional Endodontic Surgery. However, if this is true for both single and multi-rooted teeth is not answered. The results showed the need for large-scale randomised trials.

David Levenson

NYU College of Dentistry, New York, USA

1. Kim S, Kratchman S. Modern endodontic surgery concepts and practice: a review. J Endod 2006; 32: 601-623.

Evidence-Based Dentistry (2012) 13, 108. doi:10.1038/sj.ebd.6400892 